\title{
POSTERS
}

\section{An Empirical Analysis of the Stellar Wind and Planetary Nebulae of the [WC10] Central Stars CPD-56 8032 and He 2-113.}

\author{
Orsola De Marco, M.J. Barlow and P.J. Storey
}

Dept. of Physics and Astronomy, University College London, Gower Str., London WC1E 6BT, UK

[WC] stars are $\mathrm{H}$-deficient central stars of PN which have developed a dense, fast stellar wind. Their spectra can mimic the spectra of massive $\left(\mathrm{M}_{\mathrm{in}} \sim 50 \mathrm{M}_{\odot}\right)$ Wolf-Rayet stars of the carbon sequence. Deriving their parameters is of importance both in understanding the PN and the Wolf-Rayet phenomena. Spectra of both objects were obtained at the AAT in May 1993 with the UCL Echelle Spectrograph (3500-9200 $\AA, R=50,0000$ ). The reddening, determined from comparing the $\mathrm{H} \beta$ nebular line fluxes with radio fluxes from Purton et al. (MNRAS 128, 321, 1982), yielded $E(B-V)=0.68$ (CPD-56 8032) and 1.00 (He 2113). Distances are derived using two different methods and they agree within the relative uncertainties. They are 1.35 and $1.50 \mathrm{kpc}$ for CPD-56 8032 and $\mathrm{He} 2-113$, respectively. The electron temperatures of the $\mathrm{C}$ II line formation region in the wind is derived from a recombination line analysis (20,000 and 17,000 K for CPD-56 8032 and $\mathrm{He} 2-113$, respectively). From the same stellar wind recombination line analysis, we find $\mathrm{C} / \mathrm{He}=0.12$ and 0.16 , together with $\mathrm{O} / \mathrm{He}=0.19$ and 0.25 , for $\mathrm{CPD}-56^{\circ} 8032$ and $\mathrm{He} 2-113$ respectively.

Nebular temperatures ( $8800 \mathrm{~K}$ for CPD-56 8032 and $8400 \mathrm{~K}$ for $\mathrm{He} 2-113$ ), densities $\left(\log \left(\mathrm{N}_{e}\right)=4.8 \mathrm{~cm}^{-3}\right.$ for both objects) and abundances of $\mathrm{N}, \mathrm{S}$ and $\mathrm{C}$, are also obtained. N/H and $\mathrm{S} / \mathrm{H}$ have solar values while $\mathrm{C} / \mathrm{H}$ is considerably enhanced. Pre-COSTAR HST H $\beta$ images were deconvolved and are presented below for the first time. 


\section{Central Stars}
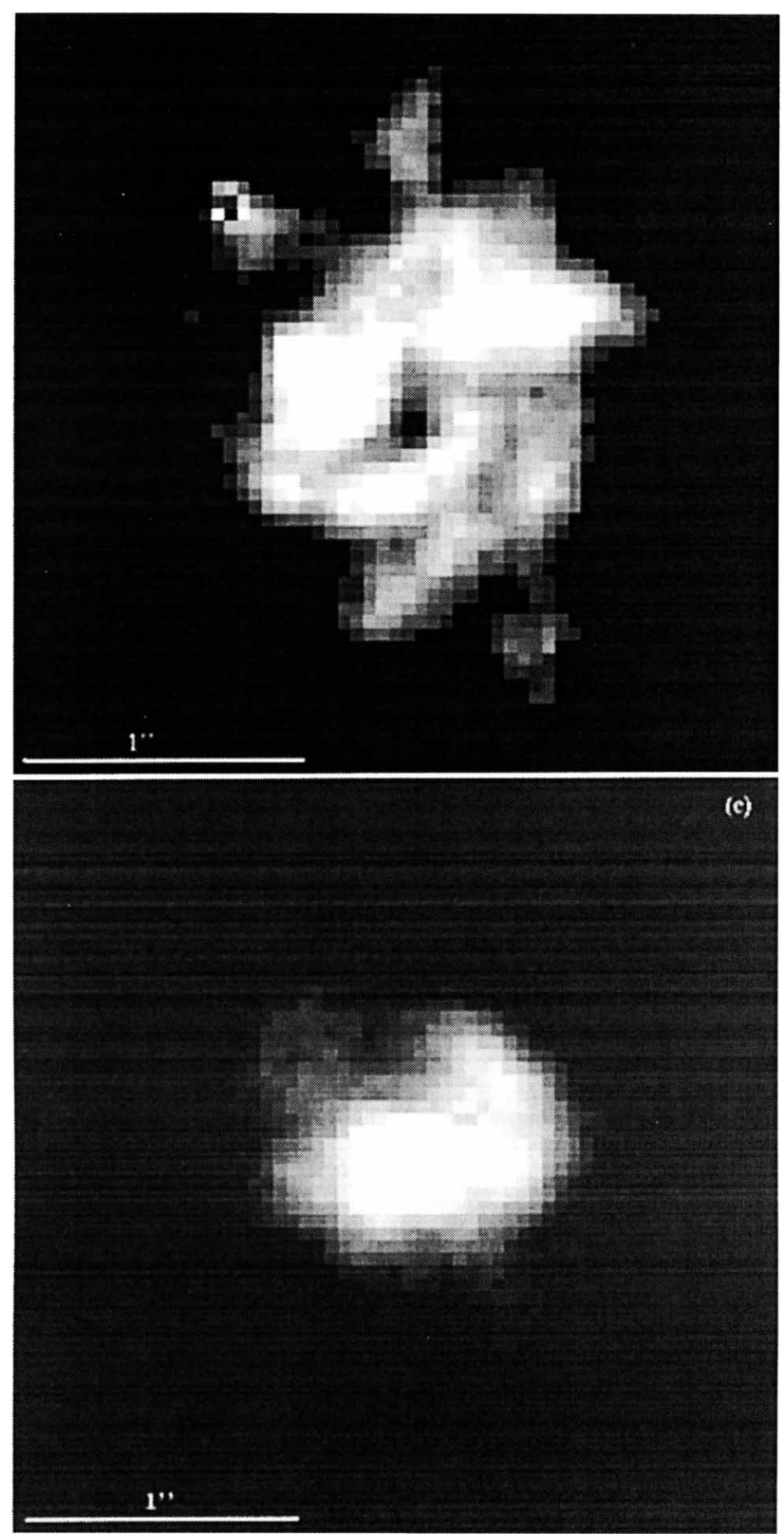

Figure 1: HST WFPC-1 images of the nebulae around CPD-56 8032 (left) and He 2-113 (right). North is towards the upper left-hand corner in each case. 